\title{
Asociación Sindromática: Poland, Goldenhar, Moebius, Klippel-Feil. Presentación de un Caso Clínico
}

\author{
CAROLINA CARES B. ${ }^{1}$, TERESA ARAVENA C. ${ }^{2}$ \\ 1. Becaria Genética Clínica, Hospital Clínico Universidad de Chile. \\ 2. Genetista Clínico, Sección Genética Clínica, Hospital Clínico Universidad de Chile. Jefe Unidad de Genética, \\ Sección Genética Clínica Hospital Dr. Sótero del Río.
}

\begin{abstract}
Poland, Goldenhar, Moebius, Klippel-Feil Syndrome. A Clinical Case

Poland Syndrome is a congenital defect characterized by a unilateral absence of the clavicular and sternocostal portion of the pectoralis muscles associated to abnormalities of other muscles of the thoracic wall, ribs, breast and upper extremity. It is found in one of 20 to 32 thousand newborns. It is found sometimes associated to other syndromes, most often with Moebius Syndrome, and rarely with Goldenhar and Klippel-Feil. Due to the association, a common pathogenic cause has been postulated, that being an anomaly of vascularization during embrionary development. Clinical Case: A newborn male was seen who presented with Poland, Goldenhar, Moebius and Klippel-Feil Syndromes. Clinically, he presented left hemifacial mycrosomy, microtia, shortening and paralysis of the facial nerve; his neck was short and movement was limited due to C4-C5 fusion; agenesis of left pectoralis, hypoplasia of left radius and hand. There was no known additional family cases, being thus, a sporadic syndromatic association.

(Key words: Syndromatic association, Poland Syndrome, Goldenhar Syndrome, Moebius Syndrome, KlippelFeil Syndrome).

Rev Chil Pediatr 2010; 81 (1): 53-57
\end{abstract}

\section{RESUMEN}

Introducción: El síndrome de Poland es un defecto muscular congénito, heterogéneo, caracterizado por ausencia unilateral de las porciones clavicular y/o esternocostal del músculo pectoral mayor, que se puede asociar a compromiso de otros músculos de la pared torácica, costillas, mama y extremidad superior. Se presenta con una frecuencia entre 1/20 000 a 1/32 000 nacidos. El síndrome de Poland se presenta en algunas ocasiones asociado a otros síndromes, siendo clásica con el síndrome de Moebius. Excepcionalmente se ha descrito la aparición conjunta con otros síndromes como Goldenhar y Klippel-Feil. Por la relación que existe entre ellos se plantea una patogenia común: anomalía en la vascularización, durante el desarrollo embrionario. Caso

Trabajo recibido el 08 de septiembre de 2009, devuelto para corregir el 05 de octubre de 2009, segunda versión el 22 de octubre de 2009, aceptado para publicación el 03 de noviembre de 2009. 
Clínico: Paciente de sexo masculino, con asociación sindromática de Poland, Goldenhar, Moebius y KlippelFeil. Como características clínicas presenta a izquierda microsomía hemifacial, microtia, acortamiento de rama mandibular y parálisis facial; cuello corto y limitación de movimientos por fusión de C4-C5; agenesia del pectoral mayor izquierdo, hipoplasia de radio y mano izquierda. Sin antecedentes familiares, se trataría de un caso esporádico de asociación sindromática.

(Palabras clave: Asociación sindromática, Síndrome de Poland, Síndrome de Goldenhar, Síndrome de Moebius, Síndrome de Klippel-Feil).

Rev Chil Pediatr 2010; 81 (1): 53-57

\section{Introducción}

El síndrome de Poland (SP) fue descrito por primera vez en 1841 por Alfred Poland, quien realizó una autopsia a un conscripto de 27 años de edad, la que reveló la ausencia del pectoral menor y la hipoplasia de la porción esternocostal del pectoral mayor, serrato anterior y oblicuo abdominal externo, además de hipoplasia ipsilateral de la mano con sindactilia de los cuatro dedos a nivel de las articulaciones interfalángicas, con agenesia de tres de las falanges medias e hipoplasia de la cuarta. Los vasos sanguíneos que irrigan los espacios intercostales se describieron como muy pequeños ${ }^{1}$.

El espectro de los defectos torácicos en el síndrome de Poland incluye un amplio rango de anomalías, desde hipoplasia a aplasia de mamas y costillas, depresión de la pared torácica, anomalías esternales, ausencia de vello axilar y poco desarrollo de grasa subcutánea. Las extremidades superiores pueden estar indemnes o severamente afectadas, semejando una focomelia. La deformidad de la mano clásica incluye sindactilia, grado variable de braquidactilia, y severa hipoplasia o aplasia de las falanges medias ${ }^{2}$.

Se ha reportado la asociación de SP con otras anomalías del desarrollo como el síndrome de Moebius (parálisis facial congénita) por Parker et al 1981, síndrome de Goldenhar (microsomía hemifacial) por Cobben et al 1992, y síndrome de Klippel-Feil (fusión congénita de vértebras cervicales) por Erol et al 2004.

Se presenta el caso clínico de un paciente con una asociación sindromática, afectado por los síndromes de Poland, Moebius, KlippelFeil y Goldenhar. Como se mencionó existen reportes de casos de asociaciones sindromáti- cas, pero no de la presencia de los cuatro síndromes en un solo paciente.

El objetivo de esta publicación es dar a conocer la posibilidad de que un paciente presente simultáneamente todos estos síndromes, sugiriendo un mecanismo etiopatogénico común para ellos.

\section{Caso clínico}

Paciente de sexo masculino, de 10 años de edad, hijo único, padres no consanguíneos, sin antecedentes familiares de importancia. Embarazo controlado, sin patologías ni contacto con teratógenos. Parto vaginal de 40 semanas. RNT, AEG. Antropometría al nacimiento: peso $3120 \mathrm{~g}$, talla $49 \mathrm{~cm}$, perímetro cefálico 34 cm y Apgar: 8-9. Al examen físico destacaba una microsomia hemifacial izquierda (figura 1), microtia izquierda severa (figura 2), acortamiento de la rama mandibular izquierda, parálisis facial periférica izquierda. Cuello corto y ancho con limitación en movimientos laterales (figura 3). Tórax con agenesia del músculo pectoral mayor izquierdo con asimetría torácica (figura 4). En las extremidades presentaba agenesia del radio izquierdo y pulgar de mano izquierda (figura 1). Presentaba además hipoacusia severa de oído izquierdo secundario a microtia severa y desarrollo intelectual normal. Todo el resto de órganos y sistemas no presentan afectación.

Dentro de los exámenes destacaba una radiografía de columna que mostró hemivertebras cervicales $\mathrm{C} 3-\mathrm{C} 4$, fusión de $\mathrm{C} 4-\mathrm{C} 5$; cariograma $46, \mathrm{XY}$.

El diagnóstico del paciente fue de una asociación sindromática: Síndrome de Poland, Síndrome de Moebius, Síndrome de Goldenhary y Síndrome de Klippel-Feil. 


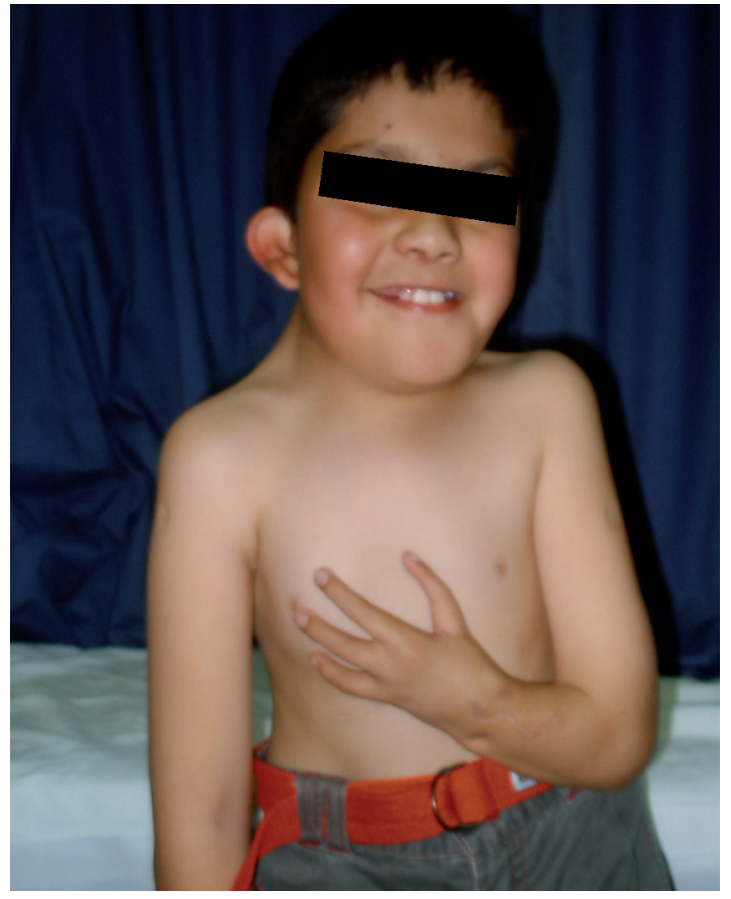

Figura 1. Microsomía hemifacial con parálisis facial izquierda. Agenesia de radio y pulgar.

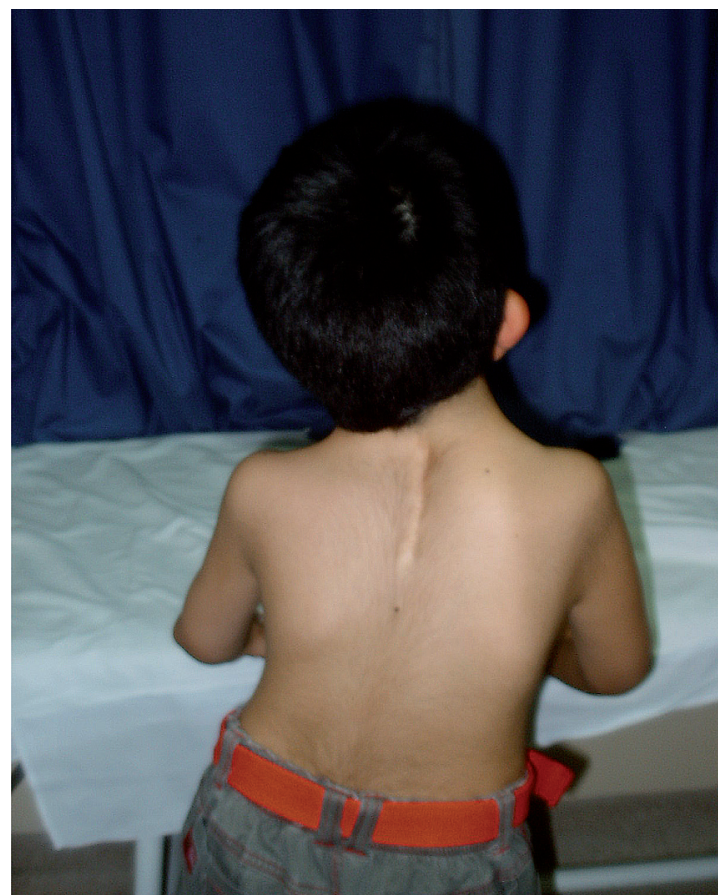

Figura 3. Cuello corto con fusión de C4-C5 y hemivértebras cervicales.

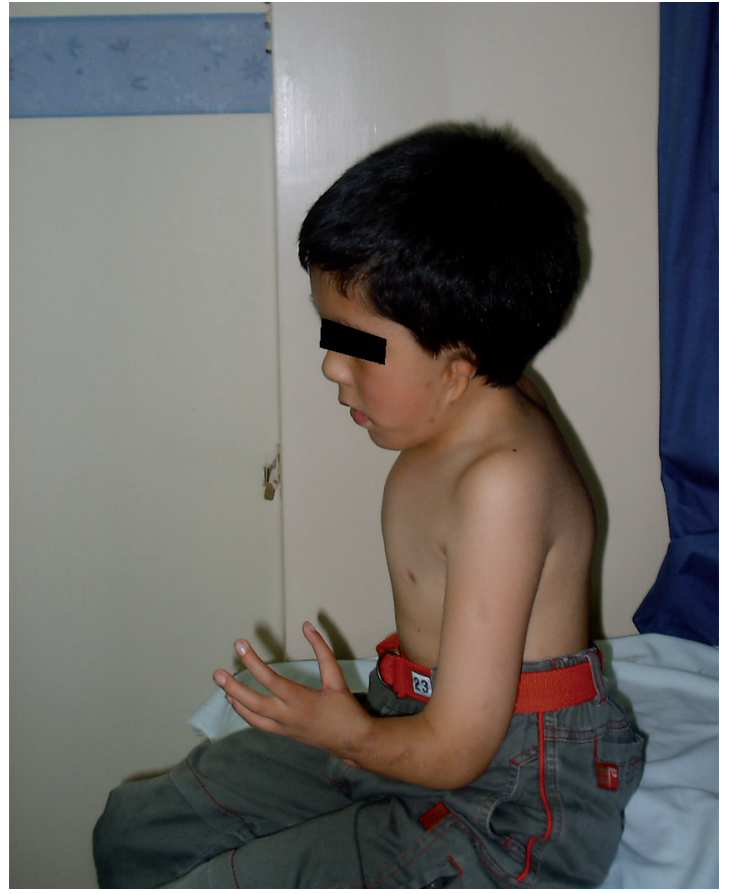

Figura 2. Microtia.

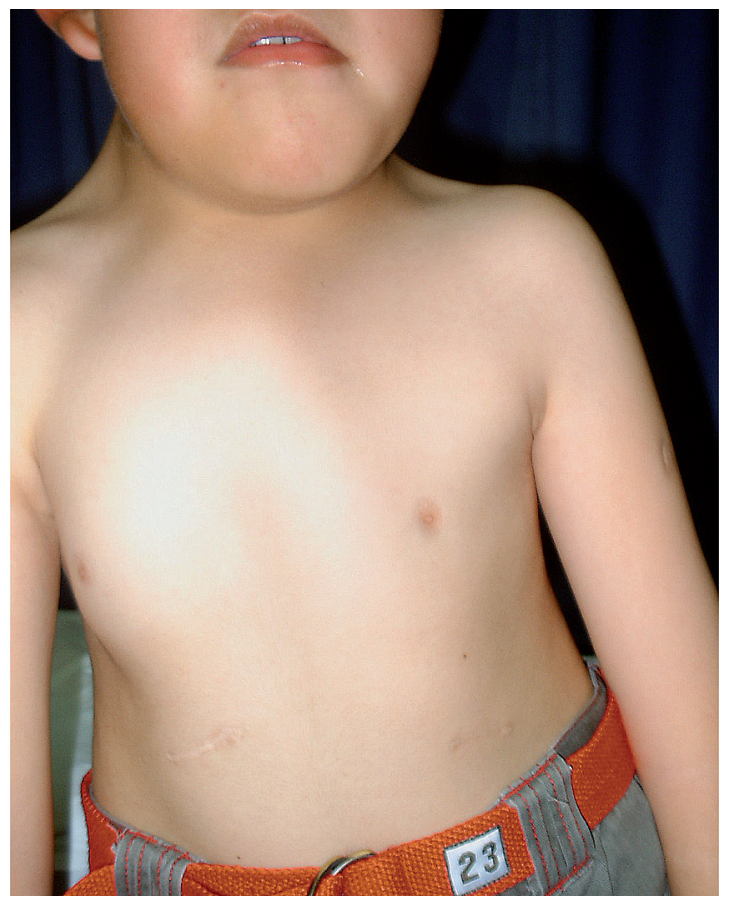

Figura 4. Agenesia del Pectoral mayor, con asimetría torácica. 


\section{Discusión}

El síndrome de Poland, es la anomalía del músculo pectoral más frecuente, con una incidencia de 1:20 000-30 000 RNV. El SP es más común en hombres que en mujeres y es más frecuente en el lado derecho que en el izquierdo ${ }^{1-3}$. La definición antigua de SP incluía la combinación de la ausencia o hipoplasia de los músculos pectoral mayor y/o menor con anomalías esqueléticas, cutáneas y subcutáneas en el hemitórax ipsilateral y grados variables de defectos en las extremidades superiores. Sin embargo, esta definición fue modificada por Al-Qattan en el $2001^{4}$ cuando el consideró la hipoplasia o aplasia del músculo pectoral como una forma moderada de SP. La severidad y extensión del SP varía ampliamente entre los individuos afectados ${ }^{2}$. La mayoría de los casos de SP reportados son esporádicos, sin embargo, ocasionalmente se han reportado casos familiares. Estos casos han mostrado gran variabilidad intrafamiliar ${ }^{5-8}$. Junto con esto se han reportado a la vez asociación del síndrome de Poland con otros defectos del desarrollo como el síndrome de Klippel-Feil, que consiste en la segmentación de la columna cervical, defecto que secundariamente causa la tríada de cuello corto, inserción posterior del pelo baja, cuello ancho con limitación de los movimientos cervicales ${ }^{9}$.

Otra asociación reportada, es con el síndrome de Moebius que consiste en la parálisis unilateral o bilateral del nervio facial, comúnmente con oftalmoplejía externa, por la paresia del nervio abductor ${ }^{10}$. También se ha reportado la asociación con el síndrome de Goldenhar o facio-auriculo-vertebral, que presenta múltiples alteraciones: a nivel facial hipoplasia de las regiones malar, mandibular y/o maxilar del lado afectado, microtia con diversas repercusiones auditivas, alteraciones oftalmológicas como tumores epibulbares, microftalmia y estrabismo entre otros ${ }^{11}$.

La etiología del SP es desconocida, sin embargo, se han planteado algunas teorías. Dentro de ellas destaca la que hace mención a la irrigación de la región pectoral y las estructuras adyacentes, en la embriogénesis. El músculo pectoral mayor se diferencia en la sexta semana de desarrollo embrionario. La porción esternocostal del músculo, y el tejido que cubre la mama junto con parte de la pared anterior del tórax, reciben el flujo sanguíneo desde la arteria torácica interna. Una interrupción del flujo de esta arteria en un período crítico del desarrollo, antes del desarrollo de circulación colateral, puede producir una isquemia y la posterior pérdida del desarro1lo y/o degeneración de estas estructura ${ }^{12}$. En 1986 Bouwes-Bavinck y Weaver, acuñaron el término SASDS (disruption subclavian artery supply sequence) como hipótesis para explicar la asociación sindrómica de Poland, KlippelFeil y Moebius, proponiendo una causa común para las diversas afecciones que comprometen la región irrigada por la arteria subclavia en el período embrionario. Esto sugiere que entre la $4^{\mathrm{a}}$ y la $6^{\mathrm{a}}$ semana de gestación, la alteración de diversas ramas de la arteria subclavia puede generar la regresión prematura y obstrucción que conducen a la devascularización, denervación, fibrosis y atrofia del segmento afectado ${ }^{12}$. El caso clínico presentado, con la coexistencia en un mismo paciente de todos los síndromes, sugiere una etiopatogenia común, que podría estar dada por la alteración de la arteria subclavia, posiblemente en un período temprano de la embriogénesis, de tal manera que varias de sus ramas estarían afectadas, generándose así un fenotipo altamente afectado.

\section{Referencias}

1.- Lord M, Laurenzano K, Hartmann R: Poland's Syndrome. Clinical Pediatrics, 1990; 29: 606-9.

2.- Baban A, Torre M, Bianca S, Buluggiu A, Rossello M, Calevo $M$, ValleM, et al: Poland syndrome with bilateral features: case description with review of the literature. Am J Med Genet A 2009; 149A: 1597-602.

3.- Jones $H$ : Congenital absence of the pectoral muscles. $\mathrm{Br}$ Med J 1926; 6: 59-60.

4.- Al-Qattan M: Classification of hand anomalies in Poland's syndrome. Br J Plast Surg 2001; 54: 132-6.

5.- Cobben JM, Robinson PH, van Essen AJ, van der Wiel $H L$, ten Kate LP: Poland anomaly in mother and daughter. Am J Med Genet 1989; 33: 519-21.

6.- Darian VB, Argenta LC, Pasyk KA: Familial Poland's syndrome. Ann Plast Surg 1989; 23: 531-7.

7.- Rojas-Martínez A, García-Cruz D, Rodríguez-García A, 
Sánchez-Corona J, Rivas F: Poland-Moebius syndrome in a boy and Poland syndrome in his mother. Clin Genet 1991; 40: 225-8.

8.- Shalev SA, Hall JG: Poland anomaly-report of an unusual family. Am J Med Genet 2003; 118A: 180-3.

9.- Issaivanan $M$, Verinderjit $S$, Virdi, Veena R: Palmar, Subclavian Artery Supply Disruption Sequence- Klippel Feil and Mobius Anomalies. Indian J Pediatr 2002; 69: 441-4.
10.- Kumar D: Mobius syndrome. J Med Genet 1990; 27 : 122-6.

11.- Iier: Instituto de Investigación de enfermedades raras. Síndrome de Goldenhar. http://iier.isciii.es/er/prg/ er_bus2.asp?cod_enf=1151. Última visita 27-07-2009.

12.- Bouwes Bavinck JN, Weaver DD: Subclavian artery supply disruption sequence: Hypothesis of a vascular etiology for Poland, Klippel-Feil, and Moebius anomalies. Am J Med Genet 1986; 23: 903-18. 